\title{
STROKE - A SYNDROME, NOT A DIAGNOSIS
}

\author{
HABIB $M^{1}$
}

Very frequently, we fail to recognise that stroke is not a single diagnosis, rather a syndrome and includes completely distinct three separate diseases. These are Ischaemic stroke (ISt), Intracerebral haemorrhage (ICH) \& Subarachnoid haemorrhage (SAH). They differ in pathogenesis, risk factors, presentation, and most importantly treatment \& prognosis. Stroke, in general, affect $1-4$ per thousand populations $^{1}$, where ISt is $80 \%$, ICH $15 \%$ and SAH $5 \%$. In acute hospital settings, the scenario is different and ICH predominates over ISt because, ICH occurs in relatively younger patients and causes dramatic presentation with headache, vomiting, impaired consciousness and convulsion requiring urgent hospitalisation. Presentations common to both ISt $\&$ ICH are weakness of one side of the body (hemiparesis) \& difficulty in speech (dysarthria/dysphasia) and most of the ISt patients present with just these features $\&$ many never seek acute hospital admission. On the other hand, SAH is a devastating disorder presenting with acute severe headache \& unconsciousness and often without any lateralised complains.

The clinico-pathogenetic definition of stroke by World Health Organisation (WHO) since $1980^{2}$ has become impractical in last decades. This is largely because of advent of sophisticated neuro-imaging techniques and introduction of thrombolysis in acute ISt due to cerebral infarct (CIf). The main limitation of WHO definition is the 24 hour time required for diagnosis of stroke from the onset of event. This used to be necessary to differentiate cerebral transient ischaemia (TIA) and completed stroke (infarct or haemorrhage). With the introduction of CT scan \& MRI, it is now possible to confirm infarct or haemorrhage within minutes of the event. It is then possible to thrombolyse selective cases of ISt, the window period for intervention of which is only 3-6 hours ${ }^{3}$. This is why the working definition of stroke now includes neuroimaging findings and is defined as "sudden onset of focal (rarely global) neurological dysfunction of nontraumatic cerebral vascular origin that lasts more than 24 hours or the patient dies within this period or neuroimaging shows an acute clinically relevant lesion" ${ }^{4}$. If the patient recovers completely within this period and neuroimaging is negative for any relevant lesion, it is called TIA.

ISt is the commonest of the stroke syndrome and is due to occlusion of cerebral vessel as a result of one of a number of causes including atherosclerosis, embolus, arteritis \& hyperthrombotic state. On the other hand, ICH is due to rupture of cerebral vessel as a result of weakness of vascular wall due either to hypertensive aneurysm, congenital AVM, or amyloid angiopathy. SAH is caused by rupture of congenital weakness of vessel wall causing aneurysm.

Regarding risk factors, all the well known modifiable \& treatable factors namely hypertension (HTN), diabetes mellitus, smoking, dyslipidaemia, IHD, AF, cardiac valvular \& septal defects are mainly risk factors for ISt. Only, HTN and smoking are found to be strongly relevant for ICH and weakly related to SAH bleeding 5 .

The acute management of stroke syndrome depends on the specific type of stroke, and prevention/treatment of acute complications. The critical decision is to make the specific diagnosis and start antithrombotic therapy in ISt as soon as possible i.e. antiplatelet in noncardioembolic and anticoagulant in

1. Prof Mansur Habib, Professor \& Head, Department of Neurology, Dhaka Medical College \& Hospital 
cardioembolic cases. General supportive treatment, judicial management of hypertension \& diabetes are essential. Preventive strategies for acute complications and treat them as they happen is vital in the acute stage. These complications in the acute stage of stroke are aspiration, infection, electrolyte imbalance, deep vein thrombosis $\&$ pressure sores. These can cause more debility to the patient than the stroke itself. Prophylactic anticonvulsants and dexamethasone in absence of clinical evidence of raised intra-cranial pressure (ICP) are not recommended and can cause more harm than good. Nimodipine is necessary in $\mathrm{SAH} \& \mathrm{ICH}$ with ventricular extension to prevent vasospasm and subsequent neurological deficit. In $\mathrm{SAH}$, it is important to investigate the patients with cerebral angiography to detect aneurysm and intervention may be necessary to secure it, to prevent rebleed. Very few cases of ISt \& ICH may require surgical intervention in specified selective indications where large ICH or CIf causes raised ICP \& rapidly progressive clinical deterioration despite adequate medical conservative treatment. Physiotherapy is crucial and needs to be started early in ISt when the patient becomes stable ${ }^{6}$ but differed for 2 weeks in ICH due to increased chance of rebleed during this time.

After the acute treatment, it is important to look into the long term management that includes secondary prevention strategies, continuation of physiotherapy and rehabilitation. Regarding secondary prevention, continuation of antithrombotic therapy in ISt and adequate treatment of risk factors for all types of strokes are essential. This requires lifetime treatment plan with periodic follow-ups.
Lastly, prognosis of stroke syndrome again depends on its type, extent of brain damage, occurrence of complications and health status of the patient prior to stroke. In general, $\mathrm{ICH} \&$ SAH carries more mortality than ISt and, ISt carries more long term morbidity than ICH \& SAH.

In conclusion, it cannot be emphasised enough that from the very beginning of presentation, stroke as single diagnosis should be avoided and a specific diagnosis of either ISt, ICH or $\mathrm{SAH}$ is made for better management of the patients.

J Dhaka Med Coll. 2015; 24(1) : 1-2.

\section{References:}

1. Mohammad QD, Habib M, Hoque A et al. Prevalence of stroke above forty years. Mymensingh Med J. 2011 Oct; 20(4):640-4.

2. Aho K, Harmsen P, Hatano S, Marquardsen J, Smirnov VE, Strasser T. Cerebrovascular disease in the community: results of a WHO collaborative study. Bull World Health Organ. 1980; 58: 113130.

3. William JP, Colin P et al.2015 AHA/ASA Focused Update of the 2013 Guidelines for the Early Management of Patients With Acute Ischemic Stroke Regarding Endovascular Treatment. A Guideline for Healthcare Professionals from the American Heart Association/American Stroke Association. Stroke. 2015; 46:000-000. DOI: 10.1161 /STR.0000000000000074.

4. Ralph L. S, Scott E. K, Joseph P. B et al. AHA/ ASA Expert Consensus Document. An Updated Definition of Stroke for the 21st Century: A Statement for Healthcare Professionals from the American Heart Association/American Stroke Association. Stroke. 2013; 44:2064-2089.

5. Martin J O, Denis X, Lisheng L et al. Risk factors for ischaemic and intracerebral haemorrhagic stroke in 22 countries (the INTERSTROKE study): a case-control study. Lancet 2010: 376; 112-23.

6. Pamela W. D, Richard Z, Barbara B et al. AHA/ ASA-Endorsed Practice Guidelines. Management of Adult Stroke Rehabilitation Care: A Clinical Practice Guideline. Stroke. 2005; 36: e100-e143. 